\title{
Snow and Cloud Detection Using Convolutional Neural Network According to the Data Derived from MSU-MR Sensor of the Spacecraft Meteor-M No. 2
}

\author{
Lubov S. Kramareva ${ }^{l}$, Alexander I. Andreev ${ }^{1}$, Evgeny V. Simonenko ${ }^{1,2}$, Egor I. Kholodov ${ }^{1}$, Julia A. \\ Shamilova ${ }^{1}$, Alexey A. Sorokin ${ }^{2}$ \\ ${ }^{1}$ Far-Eastern Center of State Research Center for Space Hydrometeorology "Planeta”, Khabarovsk, \\ Russia \\ alexander.andreev.mail@gmail.com \\ ${ }^{2}$ Shared Facility Center "Data Center of FEB RAS”, Khabarovsk, Russia
}

\begin{abstract}
The paper presents a method of forming cloud and snow masks using classifiers based on a convolutional neural network. The input data are the textures of six channels, obtained according to MSU-MR sensor in a resolution of $1 \mathrm{~km}$. A comparison was made the Random Forest algorithm, where separate pixels, NDVI and NDSI indices were used as input data as well as texture features calculated from GLCM matrix. The resulting classifiers were evaluated by calculating f-measure as well as by comparison with the results of manual interpretation by an experienced specialist and comparison with the cloud mask derived from VIIRS sensor.
\end{abstract}

Keywords: MSU-MR, snow mask, cloud mask, machine learning, convolutional neural network, CNN 


\title{
ДЕТЕКТИРОВАНИЕ СНЕГА И ОБЛАЧНОСТИ С ИСПОЛЬЗОВАНИЕМ СВЕРТОЧНОЙ НЕЙРОННОЙ СЕТИ ПО ДАННЫМ ПРИБОРА МСУ-МР КА «METEOP-M» №2
}

\author{
Л.С. Крамарева ${ }^{1}$, А.И. Андреев ${ }^{l}$, Е.В. Симоненко ${ }^{1,2}$, Е.И. Холодов ${ }^{1}$ Ю.А. Шамилова ${ }^{l}$, А.А. \\ Сорокин ${ }^{2}$ \\ ${ }^{1}$ Дальневосточный центр ФГБУ «Научно-исследовательский центр космической \\ гидрометеорологии «Планета», Хабаровск, Россия \\ alexander.andreev.mail@gmail.com \\ ${ }^{2}$ Вычислительный центр ДВО РАН, Хабаровск, Россия
}

\begin{abstract}
В работе представлен метод формирования масок облачности и снега с использованием классификаторов на основе сверточной нейронной сети. Входными данными являются текстуры, полученные по измерениям шести каналов прибора МСУ-МР с разрешением 1 км. Проведено сравнение полученного классификатора с алгоритмом Random Forest, где в качестве входных данных использовались отдельные пиксели, индексы NDVI и NDSI, а также текстурные признаки, вычисляемые из матрицы GLCM. Оценка полученных классификаторов была произведена путем расчета метрик машинного обучения на тестовом наборе текстур, таких как точность, полнота и f-мера, а также путем сравнения с результатами ручного дешифрирования опытным специалистом и сопоставлением с маской облачности по данным прибора VIIRS.
\end{abstract}

Ключевые слова: МСУ-МР, маска снега, маска облачности, машинное обучение, сверточная нейронная сеть, CNN.

\section{Введение}

Определение метеорологических и микрофизических параметров облачности и атмосферы, температуры морской поверхности, детектирование снега, мониторинг ледовой обстановки - все это далеко не полный список задач гидрометеорологии, в которых качество выходной продукции зависит от точности детектирования облачности. Задача оперативного и качественного мониторинга снежного покрова является одной из важнейших в работе гидрологов, особенно в период его схода, при этом проблема эффективного разделения снега и облачности на спутниковых изображениях до сих пор остается актуальной.

В задаче детектирования снега и облачности можно выделить два основных подхода. Первый заключается в использовании спектрального анализа значений дифференциальных индексов. Другой подход основан на применении алгоритмов машинного обучения, позволяющих рассматривать не только каждый пиксель изображения в отдельности, но и текстуры, представляющие собой участки изображения, содержащие дополнительную информацию о взаимном расположении пикселей. 
Технология машинного обучения объединяет несколько семейств алгоритмов, наиболее перспективными из которых являются искусственные нейронные сети. Последние исследования в области дистанционного зондирования Земли показывают, что нейронные сети могут успешно применяться для задач классификации подстилающей поверхности на спутниковых изображениях $[1,2]$, а также для детектирования облачности $[3,4]$, при этом использование текстурных данных повышает точность классификации по сравнению с другими методами [5].

В настоящей работе представлены классификаторы на основе сверточной нейронной сети для детектирования снега и облачности. Оценка точности классификации производилась на тестовом наборе текстурных данных с использованием показателя f-меры. Получаемые с помощью классификаторов маски прошли визуальную проверку по результатам ручного дешифрирования спутниковых изображений. Кроме того, произведено сравнение получаемой маски облачности с аналогичным продуктом по данным прибора VIIRS KA «Suomi NPP», показавшее достаточно высокий уровень качества представленных классификаторов.

\section{Формирование обучающего и тестового наборов данных}

В представленной работе задача классификации решается методами на основе машинного обучения с использованием предварительно собранных наборов данных, включающих образцы различных типов поверхностей с указанием их принадлежности к определенному классу.

Учитывая, что снег и облачность имеют схожие спектральные характеристики, их разделение при помощи пороговых значений на больших территориях сильно затруднено. Одним из вариантов решения этой проблемы является использование текстур, размер которых подбирается экспериментально в зависимости от пространственного разрешения съемки, точности классификации, скорости работы алгоритма и прочих условий. Применительно к решаемой в работе задаче размер текстур определен как $32 \times 32$ пиксела изображения, включающего шесть каналов с длинами волн 0,62, 0,86, 1,72, 3,8, 11 и 12 мкм. В наборе текстур использовались изображения территории России, полученные в дневное время суток по данным прибора МСУ-МР, установленного на космическом аппарате «Метеор-М» №2. Данный прибор производит съемку земной поверхности с разрешением 1 км в надире.

На рисунке 1 приведены примеры текстур, полученные в результате RGB синтеза, где красному каналу соответствует длина волны 0,62, зеленому - 0,86 и синему - 1,72 мкм. Текстуры могут включать в себя как один класс (рисунки 1-a, 1-b, 1-c), так и сочетания классов (рисунки 1-d), при этом в центре всегда располагается классифицируемый объект. 

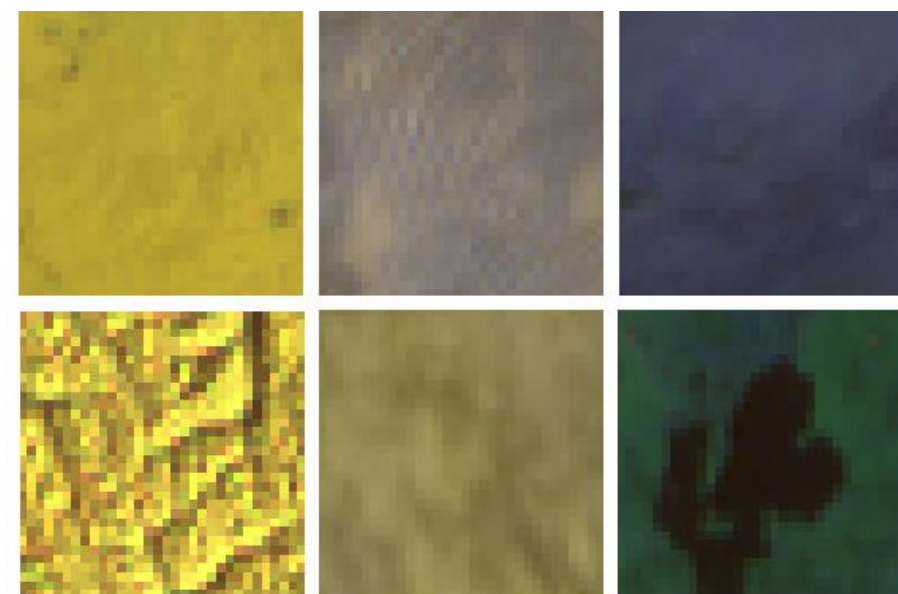

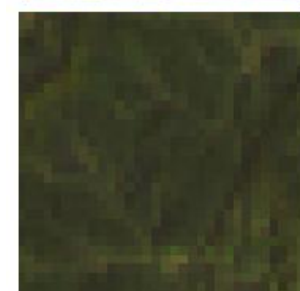

a

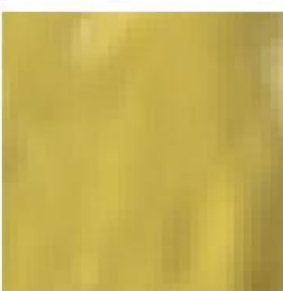

b

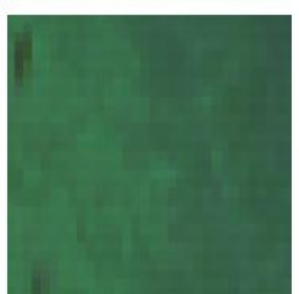

c
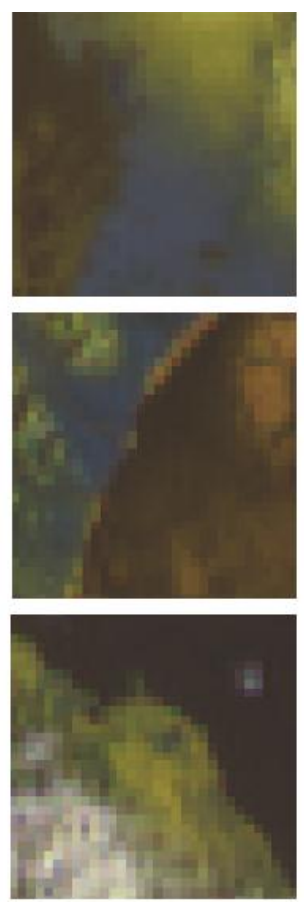

d

Рис. 1. Примеры текстур различных классов. a - снег, b - облачность, с - земля и водная поверхность, $\mathrm{d}$ смешанные классы.

В зависимости от решаемой задачи исходные классы различных типов подстилающей поверхности были сведены к трем основным для маски снега (снег, облачность и земля) и двум для маски облачности (ее наличие или отсутствие). Общий объем выборки составил приблизительно 173 тыс. текстур, включая тестовый набор данных из 42 тыс. образцов. Для улучшения качества работы классификатора и более эффективного использования набора данных, число текстур было расширено до 4,2 млн. примеров путем их поворота вокруг своей оси через каждые 15 градусов.

Полученные таким образом наборы текстурных данных, прошедшие нормализацию значений от 0 до 1 , записывались в соответствующие файлы, которые затем подавались на вход обучающего алгоритма.

\section{Сверточный нейросетевой классификатор}

Алгоритмы машинного обучения на основе сверточных нейронных сетей (Convolutional Neural Network, далее CNN) демонстрируют лучшие результаты в задачах компьютерного зрения, таких как классификация объектов на изображениях - рукописных цифр [6], лиц [7] и др. [8]. Их главной отличительной особенностью является использование операции свертки изображений, позволяющей выделять характерные для каждого объекта признаки на разных уровнях абстракции.

Для детектирования снега и облачности авторами данной статьи разработаны две соответствующие архитектуры $\mathrm{CNN}$, оптимизированные для достижения максимальной точности классификации (рисунок 2). В основу классификатора для маски снега легли идеи, использованные в архитектурах Google Inception и ResNet [9]. Классификатор для маски облачности имеет более простую архитектуру, что связано с меньшим числом классов и оптимизацией с целью ускорения его работы. 

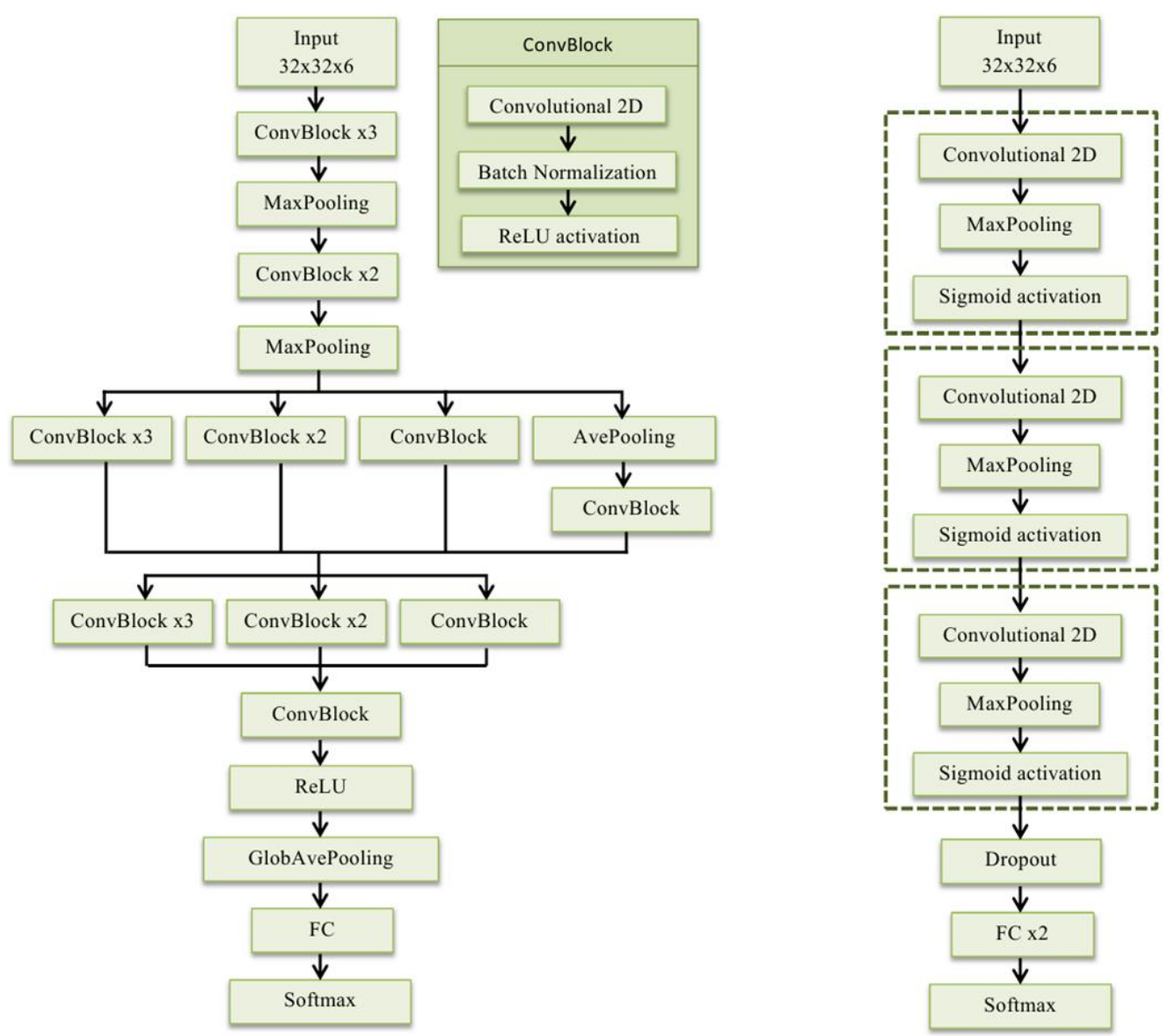

Рис. 2. Архитектуры CNN для детектирования снега (слева) и облачности (справа)

В процессе обучения каждая текстура проходит через ряд слоев свертки (Convolutional 2D на рисунке 2), в результате действия которых выделяются наиболее характерные признаки для каждого класса, причем с увеличением глубины нейронной сети повышается степень абстрактности признаков. Сами признаки кодируются при помощи сверточных фильтров, настраиваемых во время обучения. В результате свертки текстуры с этими фильтрами образуются карты признаков (feature maps) [10]. Слои субдискретизации (MaxPooling, AvePooling и GlobalAvePooling) служат для снижения размерности карт признаков.

Слои ReLU, Sigmoid activation и Softmax представляют собой функции активации каждого нейрона в слое, причем Softmax определяет принадлежность классифицируемой текстуры к одному из классов. Для моделирования функции, отражающей взаимосвязи между картами признаков и соответствующими классами, применяются слои FC (Fully Connected). Слой Dropout служит в качестве метода регуляризации [11].

\section{Оценка точности классификации}

Авторами данной работы были рассмотрены алгоритмы классификации, такие как линейный классификатор, SVM, MLP и Random Forest. Данные алгоритмы показали приблизительно одинаковую точность классификации на тестовом наборе данных, поэтому для сравнения с классификатором на основе CNN был выбран алгоритм Random Forest [15], так как он наименее требователен к входным данным и прост в настройке.

В качестве входных данных алгоритма Random Forest использовались как отдельные пиксели, по которым были рассчитаны индексы NDVI и NDSI, так и текстурные признаки, вычисляемые из матрицы GLCM [16]: 


$$
\begin{aligned}
& \text { contrast }=\sum_{i, j} C_{i, j}(i-j)^{2}, \\
& \text { dissimilarity }=\sum_{i, j} C_{i, j}|i-j|^{2}, \\
& \text { homogenity }=\sum_{i, j} \frac{C_{i, j}}{1+(i-j)^{2}}, \\
& \text { energy }=\sqrt{\sum_{i, j} C_{i, j}{ }^{2}},
\end{aligned}
$$

где $i, j$ - номер строки и столбца GLCM матрицы $C$.

Анализ результатов показал, что на классифицируемых изображениях наблюдались значительные погрешности при разделении снега и облачности, поэтому выбор был сделан в пользу классификатора на основе $\mathrm{CNN}$, показавшего более высокие результаты (таблица 1).

Таблица 1. Оценка точности работы классификатора снега на тестовом наборе данных

\begin{tabular}{|l|c|c|c|c|}
\hline \multicolumn{1}{|c|}{ Метод/входные данные } & Снег & Облачность & Земля & Среднее \\
\hline Decision Tree / пиксели & 0.89 & 0.91 & 0.94 & 0.91 \\
\hline Decision Tree / текстуры & 0.92 & 0.94 & 0.94 & 0.93 \\
\hline CNN / текстуры & 0.98 & 0.97 & 0.95 & 0.97 \\
\hline
\end{tabular}

\section{Анализ результатов}

При оценке качества получаемых классификаторами масок снега и облачности использовались следующие методики: вычисление метрик машинного обучения для тестовых текстур, сравнение с результатами ручного дешифрирования изображений оператором и сопоставление с масками облачности по данным прибора VIIRS.

Для оценки классификаторов использовалась f-мера (f-measure) - гармоническое среднее между точностью (precision) и полнотой (recall) [17]. Согласно этой метрике, вычисляемой на тестовом наборе данных, число правильных классификаций составляет не менее 97 \%.

Другой подход в оценке точности заключается в сравнении с эталонными масками, полученными в результате ручного дешифрирования оператором спутниковых изображений с использованием RGB синтеза каналов. Для сравнения были отобраны 100 полигонов на 13 спутниковых изображениях в период с января по май 2018 года. На рисунке 3 изображен результат дешифрирования облачности (b) для одного из тестовых полигонов в сравнении с маской облачности (c), полученной представленным в работе классификатором. Для полигонов была рассчитана относительная погрешность, в которой учитывалось число верных классификаций относительно эталона в каждом пикселе. Полученная таким образом относительная погрешность составила $14 \%$. 

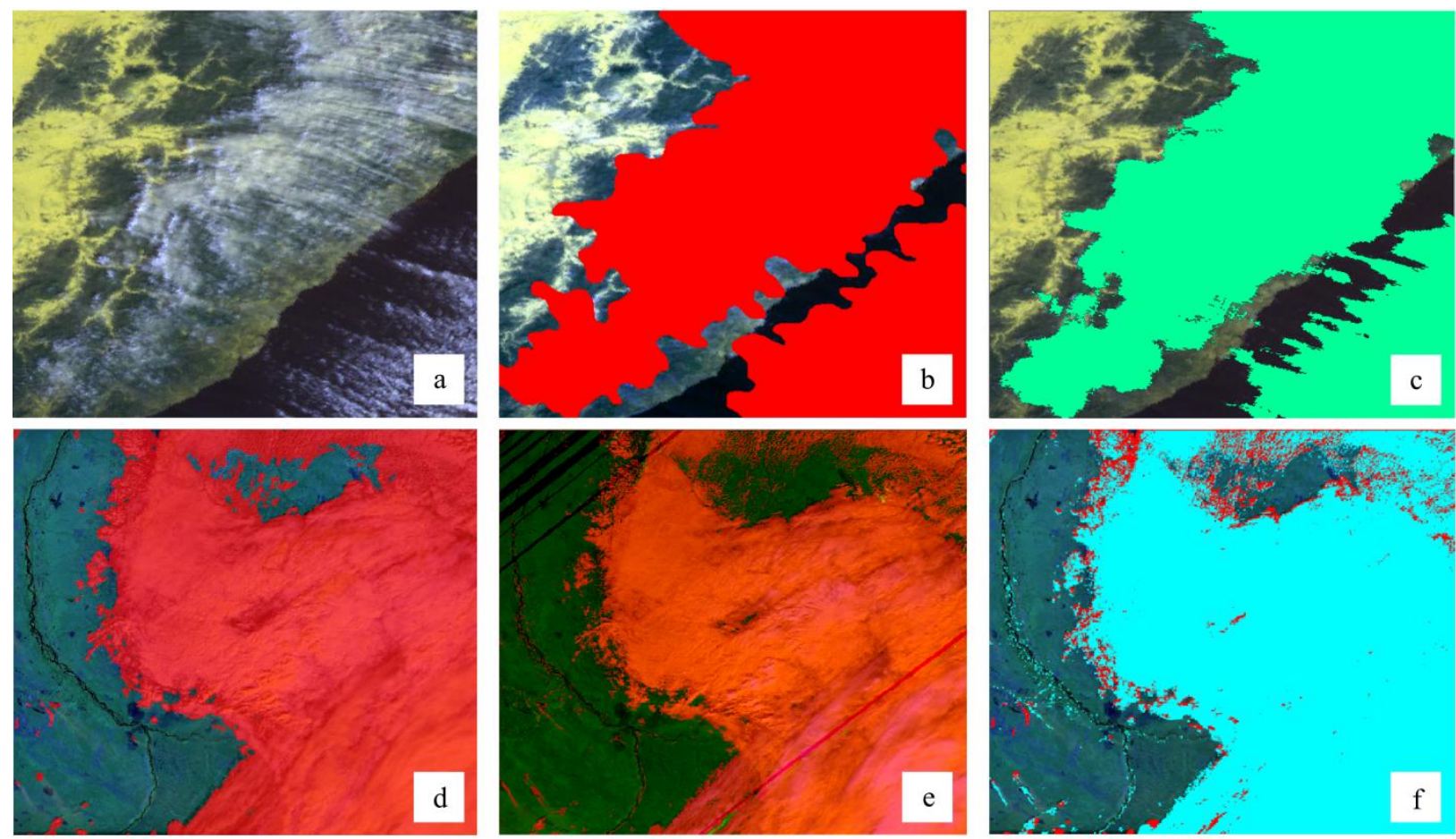

Рис. 3. Валидация маски облачности: а - исходное изображение МСУ-МР (синтез каналов 0,62, 0,86 и 1,72 мкм); $\mathrm{b}$ - результат ручного дешифрирования маски облачности; с - маска облачности, полученная классификатором, d - маска облачности по данным MCУ-MP, е - маска облачности по данным VIIRS, f сопоставление масок облачности MCУ-MP (красный цвет) и VIIRS (голубой цвет)

В дальнейшем было произведено сравнение масок облачности, получаемых классификатором, с масками по данным прибора VIIRS. Маски облачности последнего обладают достаточно высоким уровнем качества и используются в оперативной работе Дальневосточного центра НИЦ «Планета». При сравнении использовались изображения за июль и октябрь 2018 года в периоды максимального сближения спутников (разница во времени составляла не более 15 минут). Сравнение производилось путем подсчета числа облачных пикселей маски, причем в маске облачности по данным VIIRS использовались значения только уверенной облачности.

На рисунке 3 представлены маски облачности по данным приборов MCУ-MP (d) и VIIRS (e), а также результат их наложения друг на друга (f), где голубой цвет соответствует маске VIIRS, а красный - маске MCУ-MP. По результатам сравнения сделан вывод: в маске по данным МСУ-МР наблюдается значительно меньшее число ложных классификаций снега, облачности и водных объектов. Недостатком маски является неполное выделение кучевой облачности в начальной стадии развития и тонкой прозрачной перистой облачности.

\section{Заключение}

В работе представлены классификаторы на основе CNN для автоматического детектирования снега и облачности по данным прибора МСУ-МР. В качестве обучающих данных использовались текстуры с примерами подстилающей поверхности и облачности.

Результаты классификации оценивались при помощи f-меры на тестовом наборе текстур, сравнением с результатами ручного дешифрирования и маской облачности по данным прибора VIIRS. К достоинствам классификаторов на основе CNN можно отнести высокую точность детектирования облачности на фоне снега и водных объектов, а также относительно высокую помехоустойчивость. Большая часть ошибочных классификаций относится к случаям полупрозрачной перистой облачности, сквозь которую хорошо видна подстилающая поверхность.

Представленные классификаторы показали достаточно высокий уровень качества и были внедрены в оперативную работу Дальневосточного центра НИЦ «Планета». При разработке 
были использованы вычислительные ресурсы Центра Коллективного Пользования «Центр данных ДВО РАН» [18].

\section{References}

[1] Maggiori E., Tarabalka Y. Reccurent Neural Networks to correct Satellite Image Classification Maps. /In: arXiv preprint:1608.03440v3, 2017.

[2] Xiao Xiang Zhu, Devis Tuia, Lichao Mou, Gui-Song Xia, Liangpei Zhang, Feng Xu, Friedrich Fraundorfer. Deep Learning in Remote Sensing: A Review. /In: arXiv preprint: 1710.03959v1, 2017.

[3] Chen Yang, Fan Rongshuang, Bilal Muhammad, Yang Xiucheng, Wang Jingxue, and Wei Li. Multilevel Cloud Detection for High-Resolution Remote Sensing Imagery Using Multiple Convolutional Neural Networks. /In: ISPRS Int. J. Geo-Inf. 2018, 7(5), 181.

[4] Xie Fengying, Shi Mengyun, Shi Zhen Wei, Yin Jihao, and Zhao Danpei. Multi-level Cloud Detection in Remote Sensing Images Based on Deep Learning. /In: IEEE Journal of Selected Topics in Applied Earth Observations and Remote Sensing, pp(99):1-10, April 2017.

[5] Le Goff M., Tourneret J.-Y., Wendt H., and Spigai M. Deep learning for cloud detection. /In: ICPRS (8th International Conference of Pattern Recognition Systems), 2017.

[6] LeCun Y., Boser B., Denker J., Henderson D., Howard R., Hubbard W. and Jackel L. (1989). Backpropagation Applied to Handwritten Zip Code Recognition. Neural Computation, 1(4), pp.541-551.

[7] Lawrence S., Giles C., Ah Chung Tsoi and Back A. (1997). Face recognition: a convolutional neural-network approach. IEEE Transactions on Neural Networks, 8(1), pp. 98-113.

[8] Ciregan D., Meier U. and Schmidhuber J. Muli-column deep neural networks for image classification. In 2012 IEEE Conference on Computer Vision and Pattern Recognition. 3642-3649.

[9] Szegedy C., Ioffe S., Vanhoucke V. Inception-v4, Inception-ResNet and the impact of residual connection om learning. arXiv preprint arXiv:1602.07261v2, 2016.

[10] Jay Kuo C.-C. Understanding Convolutional Nerual Networks with A Mathematical Model. /In: arXiv preprint:1609.04112v2, 2016.

[11] Srivastava N. Hinton G., Krizhevsky A., Sutskever I., Salakhutdinov R. Dropout: a simple way to prevent neural networks from overfiting. - Journal of Machine Learning Research. 15 (2014), pp. 1929-1958.

[12] Diederik P. K., Jimmy Lei Ba. 2015. Adam: a method for stochastic optimization. In 2015 conference ICLR.

[13] Cilimkovic M. Neural Networks and Back Propagation Algorithm. Institute of Technology Blanchardstown, Blanchardstown Road North Dublin 15, Ireland.

[14] Nasr G. E., Badr E. A., Joun C. Cross Entropy Error Function in Neural Networks: Forecasting Gasoline Demand. Proceedings of the Fifteenth International Florida Artificial Intelligence Research Society Conference. 2002. pp. 381-384.

[15] Quinlan, J. R. (1987). "Simplifying decision trees". International Journal of Man-Machine Studies. 27 (3): 221.

[16] Haralick, Robert M., and Karthikeyan Shanmugam. "Textural features for image classification." IEEE Transactions on systems, man, and cybernetics 6, 1973.

[17] Mueller A., Guido S. An introduction to Machine Learning with Python. O'Reilly, 2017, 978-1-449-369415, p. 392.

[18] Sorokin A.A., Makogonov S.I., Korolev S.P. The Information Infrastructure for Collective Scientific Work in the Far East of Russia // Scientific and Technical Information Processing. 2017. Vol. 44. Number 4. P. 302304. 